\title{
Contemporary Management and Trends in the Treatment of Upper Tract Urothelial Carcinoma
}

\author{
Joel E. Abbott ${ }^{\mathrm{a}, \mathrm{e}}$, Arman Cicic ${ }^{\mathrm{b}}$, Anthony D. DiMatteo ${ }^{\mathrm{a}}$, Elise Fazio ${ }^{\mathrm{c}}$, \\ Julio G. Davalos ${ }^{\mathrm{d}}$
}

\begin{abstract}
Upper tract urothelial carcinoma (UTUC) represents only $5 \%$ of all urothelial cancers. The 5-year cancer-specific survival in the United States is roughly $75 \%$, with grade and stage being the most powerful predictors of survival. Nephroureterectomy with excision of the ipsilateral ureteral orifice and bladder cuff en bloc remains the gold standard treatment of the upper urinary tract urothelial cancers. However, endoscopic and laparoscopic approaches are rapidly evolving as reasonable alternatives of care depending on grade and stage of disease. A critical review of the current literature and various guidelines regarding tumor management in UTUC was undertaken, with a focus on surgical options. Topics reviewed include percutaneous and endoscopic approaches, laparoscopic nephroureterectomy (LNU), options regarding the management of the distal ureter, the role of lymphadenectomy, and the emerging role of chemotherapy in the treatment of UTUC. Both National Comprehensive Cancer Network (NCCN) and European Association of Urology (EAU) current guidelines are reviewed. Limited recommendations are provided by the American Urological Association (AUA). Scant level 1 or grade A evidence was noted in the establishment of the various guidelines. There is debate regarding how to best manage UTUC. With the current trend towards minimally invasive, localized, and precise surgical treatments for all solid malignancies, we must evaluate this movement as it applies to UTUC. Nephron sparing surgery is the preferred option, when feasible, in the management of other renal malignancies. This, too, must be considered when managing UTUC. Higher quality research is needed to better establish evidence-based guidelines. However, this is a challenging prospect given the low
\end{abstract}

Manuscript accepted for publication May 14, 2015

aDepartment of Urology, St John Providence Health, Michigan State University, 11800 E. 12 Mile Rd., Warren, MI 48093, USA

bDepartment of Urology, Metro Health Hospital, Michigan State University, 5900 Byron Center Ave. S.W., Wyoming, MI 49519, USA

${ }^{\mathrm{c}}$ Western University of Health Sciences, 309 East 2nd St., Pomona, CA 91766, USA

${ }^{\mathrm{d} C h e s a p e a k e ~ U r o l o g y ~ A s s o c i a t e s, ~ U n i v e r s i t y ~ o f ~ M a r y l a n d, ~} 806$ Landmark Dr. \#118 Glen, Burnie, MD, USA

${ }^{\mathrm{e} C}$ Corresponding Author: Joel E. Abbott, Department of Urology, St John Providence Health, Michigan State University, 11800 E. 12 Mile Rd., Warren, MI 48093, USA. Email: joel.abbott@stjohn.org

doi: http://dx.doi.org/10.14740/wjnu200w incidence of UTUC and the difficulties encountered in creating appropriate protocols.

Keywords: Transitional cell carcinoma; Endoscopic surgical procedure; Urinary tract neoplasms; Urologic neoplasms; Urinary tract diseases; Urothelium

\section{Introduction}

\section{Epidemiology}

Urothelial carcinomas (UCs) are the fourth most commonly occurring tumors [1]. Bladder tumors account for $90-95 \%$ of UCs and are the most common malignancy of the urinary tract, while upper tract urothelial carcinomas (UTUCs) account for $5-10 \%$ of UCs $[2,3]$. UTUCs account for $5-7 \%$ of all renal tumors. The estimated annual incidence of UTUC in Western countries is approximately two new cases per 100,000. Peak incidence of UTUC occurs in the eighth decade of life, and it is three times more prevalent in men than in women. The overall prognosis of UTUC depends heavily on the stage at diagnosis. Stage Ta and Tis tumors have 5-year survival rates up to $100 \%$, while 5 -year survival rates for stage T3 tumors do not exceed $40 \%$ [4]. Although rare, UTUC has a tendency for multifocality, local recurrence, and metastasis $[4,5]$. Concurrent bladder cancer is present in $17 \%$ of cases [6]. Recurrence of disease in the bladder occurs in $22-47 \%$ of UTUC patients, whereas recurrence in the contralateral upper tract is observed in 2-6\% [7]. Compared to UC of the bladder, UTUC is often more advanced at initial diagnosis with almost $60 \%$ invasive at diagnosis, presents at a higher grade, and consequently, has a poorer prognosis $[8,9]$. UTUC shares similar risk factors with bladder cancer with tobacco and occupational exposure to aromatic amines being the most common [9].

\section{Grading}

As with other solid tumors, UTUC's stage and grade are the most important prognostic factors. Before 2004, the World Health Organization (WHO) classified UTUC into three grades

Articles @ The authors | Journal compilation @ World J Nephrol Urol and Elmer Press Inc ${ }^{\mathrm{TM}} \quad$ | www.wjnu.elmerpress.com This is an open-access article distributed under the terms of the Creative Commons Attribution License, which permits unrestricted use, distribution, and reproduction in any medium, provided the original work is properly cited 

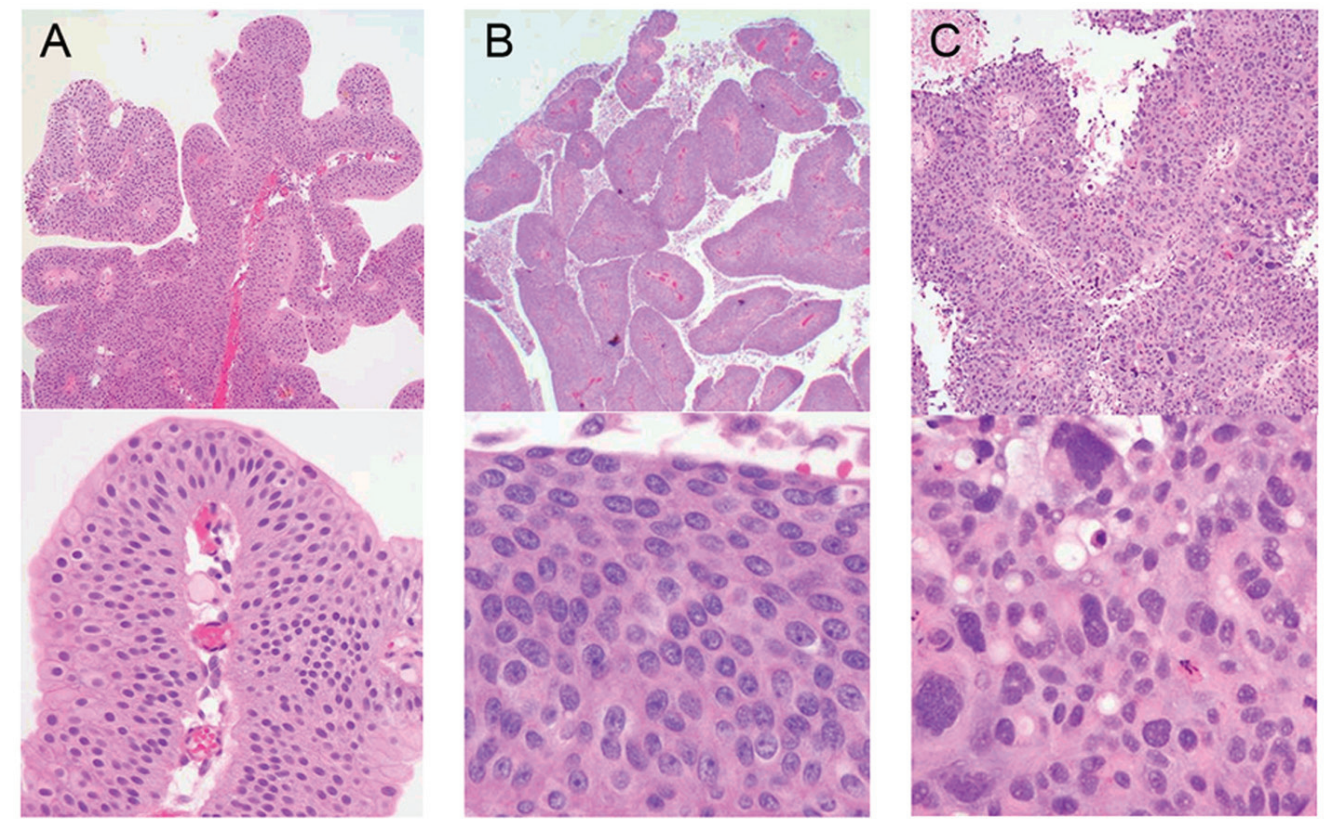

Figure 1. Low-power and high-power microscopic appearance of (A) PUNLMP, (B) low-grade urothelial carcinoma, and (C) highgrade urothelial carcinoma.

(G1, G2, and G3). Since then, the WHO classification has incorporated histological data (Fig. 1) in order to reflect rate of tumor growth, distinguishing three types of non-invasive neoplasms: papillary urothelial neoplasia of low malignant potential (PUNLMP), low-grade carcinomas, and high-grade carcinomas [3]. This classification excludes urothelial papillomas, which are completely benign.

PUNLMP is a papillary lesion with thickened urothelium in an orderly arrangement, having minimal architectural abnormalities and minimally enlarged and hyperchromatic nuclei atypia. Mitotic figures are infrequent and usually confined to the basal layer.

Low-grade papillary carcinomas have an orderly appearance, but display an easily recognizable variation of architectural and/or cytologic features, including variations of polarity and nuclear size, shape, and chromatin texture. Mitotic figures are infrequent, but may be present in the lower half of the cell layers.

High-grade papillary urothelial carcinomas have a disorderly appearance at low magnification. These tumors feature architectural abnormalities of irregularly clustered cells with disorganized epithelium, and cytologic abnormalities with pleomorphic variation. Nuclear chromatin may be clumped with prominant nucleoli. Mitotic figures, including atypical forms, are typically seen at all levels of the urothelium, and may demonstrate marked nuclear anaplasia. Histologic variants (micropapillary, clear cell, neuroendocrine, and lymphoepithelial) may be associated with the specimen in up to $25 \%$ of cases and are considered to be high grade [10].

Histologic grading of UTUC between initial biopsy and final resected pathology correlates up to $90 \%$ of the time [11]. Variation within a single lesion can be seen, however, demonstrating a spectrum of cytologic and architectural abnormali- ties. This underscores the importance of examining the entire specimen, with the highest visualized grade determining diagnosis [12]. This also brings to light a prominent challenge in UTUC research: the lack of consistent grading between physicians and investigators.

\section{Staging}

Preoperatively obtaining accurate staging and grading of an upper tract tumor is limited by contemporary biopsy techniques. Despite vast improvements in imaging, endoscopic instruments, and biopsy devices, it is difficult to routinely obtain representative tissue specimen for accurate staging that includes muscularis tissue. The information needed to accurately stage a UTUC is often available only from a post-operative specimen. This contrasts with urothelial tumors of the bladder, in which all information needed for staging is ascertained from biopsy or transurethral resection of bladder tumor.

Endoscopic biopsy cannot reliably predict upper tract tumor stage. Tumor stage has shown less than $50 \%$ correlation to final pathology, due primarily to the small size and depth potential of tissue sampling, and tumors are often upstaged after resection [12].

Urologists rely on a combination of tumor grade, endoscopic appearance, and radiologic appearance in prediction of tumor stage (Table 1). This creates some degree of variability between care providers. There has been a push for the development of criteria to better risk stratify these patients with upper tract tumors. Newer modalities have surfaced, including histologic stains and high frequency intraluminal ultrasound attempting to improve preoperative tumor staging and risk stratification [13]. 
Table 1. TNM Staging Classification for UTUC

\begin{tabular}{|ll}
\hline Primary tumor & \\
TX & Tumor cannot be assessed \\
T0 & No evidence of primary tumor \\
Ta & Papillary noninvasive tumor \\
Tis & Carcinoma in situ \\
T1 & Invasion of subepithelial connective tissue/lamina propria \\
T2 & Invasion of muscularis propria \\
T3 & Invasion of renal parenchyma or peripelvic/periureteral fat \\
T4 & Invasion of adjacent organs or through parenchyma into perinephric fat \\
Regional lymph nodes* & \\
\hline NX & Regional nodes cannot be assessed \\
N0 & Negative nodes \\
N1 & Single node $<2$ cm \\
N2 & Single node 2 - 5 cm; multiple nodes $<5 \mathrm{~cm}$ \\
N3 & Multiple nodes $>5$ cm \\
Metastasis & \\
M0 & No distant metastasis \\
M1 & Distant metastasis \\
TX & Tumor cannot be assessed \\
T0 & No evidence of primary tumor \\
\hline
\end{tabular}

${ }^{*}$ Regional lymph nodes: kidney - hilar, abdominal pera-aortic, abdominal paracaval; ureter - intrapelvic nodes. Laterality does not affect the $\mathrm{N}$ classification.

\section{Historical perspective}

In the past 15 years oncologic surgery has changed significantly as diagnostic techniques and treatment options for cancer have improved. As a result, survival and prognosis of many malignancies has improved, leading to modifications of surgical technique. Modern day malignancies are identified earlier, often as small, non-invasive lesions, and subsequent utilization of less invasive surgical techniques has yielded comparatively similar clinical results. Historically, however, UTUC presented with a more diffuse, large, multifocal, or bulky appearance, with gross hematuria often being the impetus to present. As a result, the gold standard for UTUC treatment has been radical nephroureterectomy (RNU) with removal of the ipsilateral bladder cuff. Endoscopic treatment was reserved for a very small subset of patients, including those with severe renal insufficiency, solitary kidney, or bilateral UTUC. Due to modern day advances and earlier diagnosis of lower stage tumors, laparoscopic surgery has supplanted traditional open surgical techniques, and endoscopic or segmental surgical options have taken on a greater role in management. More conservative, less invasive approaches in the management of UTUC have shown promise due to their improved perioperative morbidity, cosmesis, and earlier convalescence [14]. Despite these advantages, the efficacy and oncological safety of these laparoscopic and endoscopic procedures is a source of concern with the lack of long-term follow-up data available [15].

\section{Diagnosis}

\section{Symptoms upon initial presentation}

The most common presenting symptoms leading to diagnosis of UTUC is gross or microscopic hematuria in $56-98 \%$, flank pain seen in $20-40 \%$, and lumbar mass seen in $10-20 \%$ worldwide $[9,16]$. An additional $15 \%$ of patients do not experience symptoms of any kind and are identified following incidental abnormal imaging findings [17]. Concomitant systemic symptoms raise concern for metastatic disease and warrant an appropriate workup.

\section{Workup}

For patients presenting for evaluation secondary to hematuria, workup for UTUC proceeds in a similar fashion to cancers of the lower urinary tract. Computed tomography urography (CTU) is the gold standard imaging modality, with other options including magnetic resonance urography (MRU), and re- 
nal ultrasound $[9,16,18,19]$. Retrograde pyelograms may be included in the workup in cases of non-contrast computed tomography (CT), magnetic resonance imaging (MRI), or ultrasound when collecting system detail is deemed imperative [18, 19]. Cystoscopy should be performed as part of the hematuria evaluation $[9,16,18,19]$. If there is concern for metastatic disease, the workup should include complete blood count, basic metabolic panel, chest X-ray, and bone scan if elevated alkaline phosphatase or bone signs/symptoms present [19].

\section{General discussion of diagnostic modalities}

\section{1) Endoscopy}

Due to the multicentric nature of UC, adequate identification of all UTUC remains one of the main challenges in diagnosis [5]. Endoscopy plays the important role of visually identifying concomitant bladder tumors, additional UTUC, and aiding in the performance of biopsies. Cystoscopy, with or without ureteroscopy, and biopsy is recommended to confirm the presence of disease [16]. It is important to find all identifiable lesions in both the upper and lower urinary tracts to appropriately stage and grade the patient.

\section{2) Urine sampling}

The use of urine cytology and fluorescence in situ hybridization (FISH) in the identification of UTUC has limited value when compared to the recommended imaging modalities. Cytology and FISH are less sensitive modalities for diagnosing UTUC, but are often used for diagnostic clues. Cytology for the evaluation of the upper urinary tract should ideally be obtained in situ, in the renal cavities, and is only highly suggestive of UTUC when bladder cystoscopy is normal and carcinoma in situ (CIS) of the bladder or prostatic urethra has been excluded. These studies are more sensitive in detection of high-grade lesions. Usefulness in the setting of low-grade tumors has been disputed. Although FISH parallels its performance in bladder cancer, the preponderance of low-grade, recurrent disease in the population undergoing surveillance and minimally invasive therapy for upper tract transitional cell carcinoma (TCC) may limit its usefulness in this setting. Chen et al showed an FISH sensitivity of $41 \%$ and cytology sensitivity of $12 \%$ for low-grade upper tract tumors and concluded that until a high-sensitivity marker for low-grade urothelial cancer is developed, the surveillance of upper tract TCC will continue to require vigilant direct visual inspection [20].

Various tissue-based markers are currently being investigated as prognostic indicators in UTUC. These include cell adhesion (E cadherin and CD24), cell differentiation (Snail and epidermal growth factor receptor), angiogenesis (hypoxiainducible factor-1 alpha and metalloproteinases), cell proliferation (Ki67), epithelial mesenchymal transition (snail), mitosis (Aurora-A), apoptosis (Bcl-2 and survirin), and vascular invasion markers (recepteur d'origine nantais RON and c-met protein MET). To date, none of these markers have fulfilled the clinical and statistical criteria necessary to support their use in daily clinical decision-making, mainly as a result of disease rarity and small study sample sizes [9].

\section{3) $\mathrm{CTU}$}

Multi-phasic CTU yields the highest diagnostic accuracy for UTUC. Its sensitivity ranges from 0.67 to 1.0 and specificity ranges from 0.93 to 0.99 depending on the technique used [9].

Diagnosing UTUC using CTU is therefore preferred to MRU as a first-line test due to greater diagnostic accuracy, lower cost, and greater patient acceptability [9].

\section{4) MRU}

MRU is indicated in patients who cannot undergo CTU due to contraindications related to radiation or iodinated contrast media. Reported sensitivity for this imaging modality is 0.75 after contrast administration for tumors $<2 \mathrm{~cm}$ [21]. MRU using certain gadolinium-based contrasts is contraindicated in patients with severe renal impairment $(<30 \mathrm{~mL} / \mathrm{min}$ creatinine clearance) due to risk of nephrogenic systemic fibrosis [21].

\section{5) Ultrasound}

Detection of renal pelvis carcinoma is moderate $(82 \%)$ but sensitivities as low as $12 \%$ have been reported for the detection of UC of the ureter [22]. Ultrasound can often identify secondary signs of ureteric tumors such as hydronephrosis and hydroureter. New techniques are being investigated including intraluminal high frequency US to evaluate the depth of penetration and urothelial thickness but have yet to become mainstream.

\section{6) Ureteropyeloscopy}

Endoscopic technology has drastically improved in recent history, including better optics and smaller flexible ureteroscopes with active deflection [23]. These advances now allow routine visual surveillance of the entire urinary tract. In addressing UTUC, flexible ureteroscopy is used to visualize, biopsy, and obtain cytology in situ of the ureter, renal pelvis, and collecting system, with a technical success rate approximating 95\% [24]. Additionally, it can be used to direct conservative treatment to the identified upper tract tumor.

\section{Treatment}

\section{Radical nephroureterectomy}

In 1898, Le Dentu and Albarran were the first to describe RNU with en bloc excision of kidney and ureter in the management of UTUC. This was later modified to include complete 
removal of the ipsilateral urinary tract and a margin of bladder cuff by Kimball and Ferris in 1934 after noting high incidence (30-64\%) of residual or recurrent tumor in the short remaining ureteral segment [25]. This technique has since remained the gold standard treatment for UTUC as it meets all conditions required for successful oncological surgery: excision of the entire tumor with an adequate surgical margin, control of local recurrence, and evaluation/control of the anatomical spread of the tumor. Resection of the distal most intramural extent of the ureter, including its orifice, and a small circumferential cuff of bladder has been deemed beneficial because it is the part of the urinary tract at considerable risk of tumor recurrence [26-28]. In 1952, McDonald et al presented the "pluck" technique: primary transurethral detachment (either resection or excision) of the intramural ureter with a resectoscope and subsequent cephalad extraction ("plucking") during nephrectomy. It was not until 1995 that Palou et al emphasized the usefulness of the modified technique of utilizing transurethral resection to detach the intramural ureter $[29,30]$. Since then, several other techniques such as stripping, transurethral resection of the intramural ureter, and intussusception have been described as viable options for resection of the distal ureter. With the exception of ureteral stripping these techniques are deemed equivalent to excision of the bladder cuff [31-34].

The gold standard treatment for UTUC regardless of tumor location remains the RNU with excision of the bladder cuff. Further recommendations vary depending on the source of guidelines.

\section{European Association of Urology (EAU)}

The EAU recommends (grade B) RNU if any of the following are present: suspicious imaging findings suggesting infiltrating UTUC, high-grade tumor as demonstrated by urinary cytology, multifocality with two functional kidneys, and non-invasive, large tumors (i.e. $>2 \mathrm{~cm}$ ) [9]. RNU can be performed in an open or laparoscopic fashion, with or without lymphadenectomy, and with or without ipsilateral adrenalectomy. The EAU guidelines regarding technique of choice for RNU are as follows: bladder cuff removal is imperative (grade A); open and laparoscopic techniques are equivalent in terms of efficacy; postoperative instillation of chemotherapy is recommended after RNU to avoid bladder recurrence (grade B); lymphadenectomy is recommended in cases of invasive UTUC (grade C); and several techniques for bladder cuff excision are acceptable (grade B), except stripping [9].

\section{National Comprehensive Cancer Network (NCCN)}

NCCN guideline recommendations are organized for tumors of the renal pelvis and those of the ureter. For tumors of the renal pelvis recommendations are for RNU, regional lymphadenectomy, and consideration for neoadjuvant chemotherapy in select patients for any of the following characteristics: high-grade tumors, large tumors, or evidence of parenchymal invasion. For ureteral urothelial tumors, RNU is strictly rec- ommended for high-grade tumors of the mid ureter. All other ureteral tumors may be treated with RNU, though more conservative treatment is offered.

\section{American Urological Association (AUA)}

No specific guidelines exist for management of upper tract neoplasms. RNU with bladder cuff is acknowledged as the gold standard surgical treatment.

Ipsilateral adrenalectomy is commonly performed with RNU despite the lack of data on rates of adrenal metastasis or direct invasion by renal pelvic tumors. Huang et al evaluated the role of adrenalectomy through 110 patients with localized UTUC treated by nephroureterectomy and bladder cuff resection. Seventy of these patients underwent RNU without adrenalectomy and 40 patients were treated with concurrent adrenalectomy. There was no significant difference in 5-year survival, metastasis-free survival, or cancer-free survival between the two groups [35].

Finally, there has been little discussion in the literature concerning intrafascial vs. perifascial nephrectomy with the widely accepted idea that Gerota's fascia and perinephric fat should be excised during RNU [5].

\section{RNU vs. LNU}

Multiple sources have reported on equivalent oncologic outcomes of laparoscopic nephroureterectomy (LNU) vs. open RNU. There are over 400 observational studies, yet only one randomized clinical trial ( $\mathrm{RCT}$ ) to date analyzing laparoscopic vs. open RNU [19]. Many of these studies report improved clinical outcomes for LNU with statistically significant for decreased blood loss, postoperative pain, and mean time to discharge from hospital. However, long-term data from this study show no statistically significant difference between LNU and RNU in terms of overall 5-year cancer specific survival (89.9\% vs. $78.8 \%)$ and 5-year metastases free survival (74.4\% and $72.5 \%$ ) [19]. These findings suggest that laparoscopic techniques are better with regard to perioperative outcomes, but demonstrate long-term oncological outcomes similar to RNU [5, 9, 19].

\section{Lymphadenectomy}

There are no clear guidelines regarding the role of lymphadenectomy associated with RNU, but there are data supporting its use based on prolonged survival of patients with extended lymphadenectomy during cystectomy for urothelial bladder cancer $[5,9]$. Komatsu et al suggested a potential diagnostic role of lymphadenectomy in identifying candidates for adjuvant chemotherapy [36]. In a non-randomized, retrospective study, Miyake et al found no significant difference in survival rates between patients with and patients without concurrent lymphadenectomy [35]. However, among patients with no lymph vessel invasion, the survival rate of those with lym- 
phadenectomy was significantly higher than those without [35]. Therefore, this adjunct technique is suggested for prophylactic eradication of minimally metastatic disease $[5,35]$.

\section{Conservative surgery}

UTUC conservative treatment, also referred to as nephron sparing surgery, includes various segmental resections via endoscopic, open, or laparoscopic techniques. The following are the EAU recommendations (grade B) for conservative management of UTUC: unifocal tumor, tumor size less than 1 $\mathrm{cm}$, low-grade tumor as demonstrated by cytology or biopsy, no evidence of an infiltrative lesion on CTU, and patient understanding of close follow-up. The guidelines for choosing techniques in conservative management of UTUC are as follows (grade C): laser should be used in cases of endoscopic management; flexible ureteroscopy is preferable over rigid ureteroscopy; a percutaneous approach remains an option in small, low-grade caliceal tumors unsuitable for ureteroscopic treatment; ureteroureterostomy with wide margins is indicated for non-invasive low-grade tumors of the proximal ureter or mid-ureter that cannot be removed completely by endoscopic means, and for high-grade or invasive tumors when RSS for preservation of renal function is a goal; complete distal ureterectomy and neocystostomy with wide margins is indicated for non-invasive, low-grade tumors in the distal ureter that cannot be removed completely by endoscopic means and for highgrade, locally invasive tumors [9].

Arancibia et al, in their analysis, reported good endoscopic outcomes for single, low-grade tumors $(<15 \mathrm{~mm})$ in absence of positive urine cytology [5]. Currently accepted indications for endoscopic treatment by Mills et al are inadequate renal reserve (chronic renal impairment or solitary kidney), actual or high risk of bilateral disease (e.g. Balkan nephropathy), significant comorbidity, palliation (where cure is not possible), and papillary, superficial, low-grade disease with low invasive potential [37].

The NCCN provides less stringent guidelines for conservative therapy in treatment of UTUC. Low-grade tumors of the renal pelvis may be treated with RNU, nephron sparing procedures, or endoscopic resection with or without postsurgical intrapelvic chemotherapy or Bacillus Calmette-Guerin (BCG). Upper ureteral tumors may be treated with either RNU or endoscopic resection. Low-grade mid ureteral tumors may be treated with endoscopic resection or RNU with consideration for regional lymphadenectomy. Distal tumors of the ureter may be treated with endoscopic resection or distal ureterectomy and regional lymphadenectomy if high grade. Ureteral reimplantation is preferred in these patients, if clinically feasible [19].

\section{Ureteroscopic management}

Elliott et al suggest that endoscopic treatment in properly selected patients, specifically neodymium-yttrium-aluminumgarnet laser or electrocautery via ureteroscopy, can be safely and effectively used as a first-line treatment for UTUC. Selection criteria for an 11-year analysis of 21 renal units treated with endoscopic treatment included normal contralateral kidney, lesions with macroscopically papillary and superficial appearance, complete visualization of lesions, complete resection of lesions, lesions $<2 \mathrm{~cm}$ in diameter, no CT evidence of parenchymal invasion, and strict schedule for follow-up (based on recurrence rates of $37 \%$ and renal preservation rate of $81 \%$ ) [38].

Ureteroscopy is the preferred endoscopic treatment modality for patients with low-grade UTUC. Feasibility is contingent on patient and urologist willingness to adhere to rigorous surveillance protocols and accept repeat treatments for local recurrence [39]. Aranciba summarized the limitations of ureteroscopy, finding that the small size of equipment limits the volume of tumors treated in one procedure and often requires a second look procedure to ensure complete eradication of tumor [5].

\section{Percutaneous management}

Percutaneous access, as recommended by Adamis et al, is preferred for large tumors $(>1.5 \mathrm{~cm})$ located proximally in renal pelvis and/or upper ureter and for ureteric recurrences after a cystectomy for bladder cancer [40]. Advantages to the percutaneous technique include treatment of larger tumor volumes, access to any site in the collecting system, improved visualization, and faster resection with deeper biopsies. Percutaneous management may employ various modalities for tumor ablation, including monopolar or bipolar cautery, laser, rollerball electrode, and electrovaporization. Recurrence rates are 18$28 \%$ for low-grade tumors and approximately $50 \%$ for highgrade tumors [40].

Roupret et al recommend conservative surgical management, including ureteroscopy and percutaneous endoscopic ablation, as an alternative to open nephroureterectomy for lowgrade or superficial UTUC based on analysis of 97 patients [41]. Fifty-four of these patients underwent open nephroureterectomy, 27 underwent ureteroscopy, and 16 underwent percutaneous endoscopic ablation with a 5-year disease-specific survival rate of $81.9 \%$ for low-grade tumors and $47.3 \%$ for high-grade tumors $(\mathrm{P}=0.0001)$. Based on these results, they suggested incorporation of additional prognostic indicators, such as molecular markers, prior to implementation of conservative therapy for high-grade or invasive tumors [41].

In general, endoscopic management is not advised for high-grade tumors due to high rates of both local recurrence and disease progression. It is not advised in elective situations if pathologic analysis and tumor grade cannot be obtained [40]. High-grade (grade 3) disease recurs at higher rates independent of therapeutic modality: percutaneous 31\%, open $25 \%$; low-grade (grade 1-2): percutaneous $6 \%$, open $14 \%$. For disease-specific survival there was no difference observed between stages in patients treated percutaneously vs. open, suggesting equivalent outcomes between percutaneous and open techniques [40].

Cutress et al provided a comprehensive review of all studies evaluating endoscopic management prior to and including 
2011, including ureteroscopic ablation and percutaneous nephroscopic resection of tumor. They reported strong selection bias for favorable tumor characteristics in the endoscopically treated groups, variation in medical comorbidity, and indication for treatment across the different study groups. Biopsy verification was inconsistent and follow-up in most studies was limited to a mean of 3 years. Even though many of the studies analyzed reported a grade related risk of tumor progression and disease-specific mortality for endoscopically managed UTUC, the studies had inherent flaws that should be considered when reviewing the data [42].

\section{Summary of endoscopic management}

The goal of endoscopic management is cancer control with concurrent preservation of renal function and integrity of the urinary tract. It was initially reserved for patients with solitary kidneys or bilateral disease, but has gained acceptance in the management of small, low-stage and low-grade tumors [5]. Outcomes between RNU and nephron sparing surgery have been compared. Hall et al concluded that specific surgical procedure is an independent predictor of overall disease recurrence. In their retrospective study of 252 patients overall disease recurrence was significantly higher in patients who had parenchymal sparing surgery, distal or segmental ureterectomy, excision of tumor, and endoscopic ablation [5]. In these conservatively managed patients, understaging and undertreatment will remain a significant risk until improved minimally invasive diagnostic tools are developed. Two large cohort studies utilizing the surveillance, epidemiology, and end result (SEER) database comparing nephron sparing treatment approaches to conventional extirpative therapy showed no difference in cancer-specific survival in carefully selected patients $[43,44]$. Clear limitations have been identified in these retrospective studies, including lack of longterm follow-up. Lack of randomization limits many studies evaluating conservative UTUC management and a preference for treating the older patient with more conservative therapy has led to misleading shifts in overall survival, while cancerspecific survival has remained equivalent.

\section{Adjuvant topical agents}

Mitomycin C and BCG have long been established as efficacious adjuvant topical agents in the setting of bladder cancer. In the setting of UTUC, these agents may be employed following complete tumor eradication $[23,45]$. Current methods for delivery include retrograde instillation through a ureteric stent, antegrade instillation through a percutaneous nephrostomy tube, reflux through a double J stent, and via a reverse thermosensitive polymer plug $[23,45]$. Despite these various available modalities, difficulty still remains in isolating the agent to the upper urinary tract and reducing possible post-instillation side effects, such as ureteric obstruction and consecutive pyelovenous influx during instillation or perfusion.

Additionally, due to the rarity of UTUC and lack of RCTs assessing efficacy of local chemotherapy, there is a lack of evidence supporting its use. The medium term results are similar to those for the treatment of bladder tumors, but have not been confirmed in long-term studies [9, 40, 46]. For this reason, there are no current guidelines providing a graded recommendation on the use of adjuvant local chemotherapy in the management of UTUC [39].

Rastinehad et al gathered 20 years of data and analyzed the use of adjuvant BCG after percutaneous management of UTUC in 50 renal units and 39 control units. There were no statistical differences between the two groups when comparing recurrence, time to recurrence, and progression of disease $[5,23]$. Cutress et al reported outcomes on 18 renal units treated with adjuvant mitomycin $\mathrm{C}$ after endoscopic management, with an estimated 5-year UTUC recurrence free survival of 53.8\% for patients treated with adjuvant mitomycin $\mathrm{C}$ and $54.2 \%$ for those without adjuvant treatment $[23,42]$. To date, there is no clear evidence supporting administration of adjuvant topical therapy in the management of papillary UTUC, but reports have been limited to small, retrospective cohort studies focusing on BCG with limited follow-up of less than 36 months [23].

Although adjuvant topical therapy has not demonstrated clear efficacy for papillary UTUC, several studies have yielded positive results with upper tract CIS based on response rates, low recurrence rates, and disease-specific mortality outcomes $[23,46]$. These studies were limited, however, as diagnosis of upper tract CIS was based on positive urine cytology combined with negative radiologic imaging, rather than confirmed biopsy. Furthermore, response rates were based on restoration of normal urine cytology rather than more specific criteria, such as biopsy and ureteroscopy, and disease-specific mortality outcomes varied considerably from $9 \%$ to $40 \%$ [23].

Currently, there is no level 1 evidence supporting the use of adjuvant topical chemotherapy following conservative surgery in the treatment of UTUC. However, further investigation is warranted to assess the potential benefit of this treatment $[8$, 46].

\section{Adjuvant systemic chemotherapy and radiation}

Studies analyzing adjuvant chemotherapy and radiation in the setting of UTUC are sparse in literature, largely due to the rarity of UTUC. While immunotherapy for UTUC is often utilized in a similar fashion as lower urinary tract UC, chemotherapy and radiation are treatment modalities with ill-defined roles, especially following conservative treatment. Given the high recurrence rates associated with UTUC, adjuvant therapy has significant potential roles in UTUC management, and future randomized studies are warranted. At this time these modalities should be considered strictly experimental.

\section{Chemotherapy}

In 2012 the prospective randomized controlled perioperative chemotherapy versus surveillance in UTUC (POUT) trial began enrolling patients treated with RNU; however, the results from this investigation are pending [47]. Currently, most studies evaluating this topic are non-randomized and address adjuvant treatment following radical surgery, with mixed results 


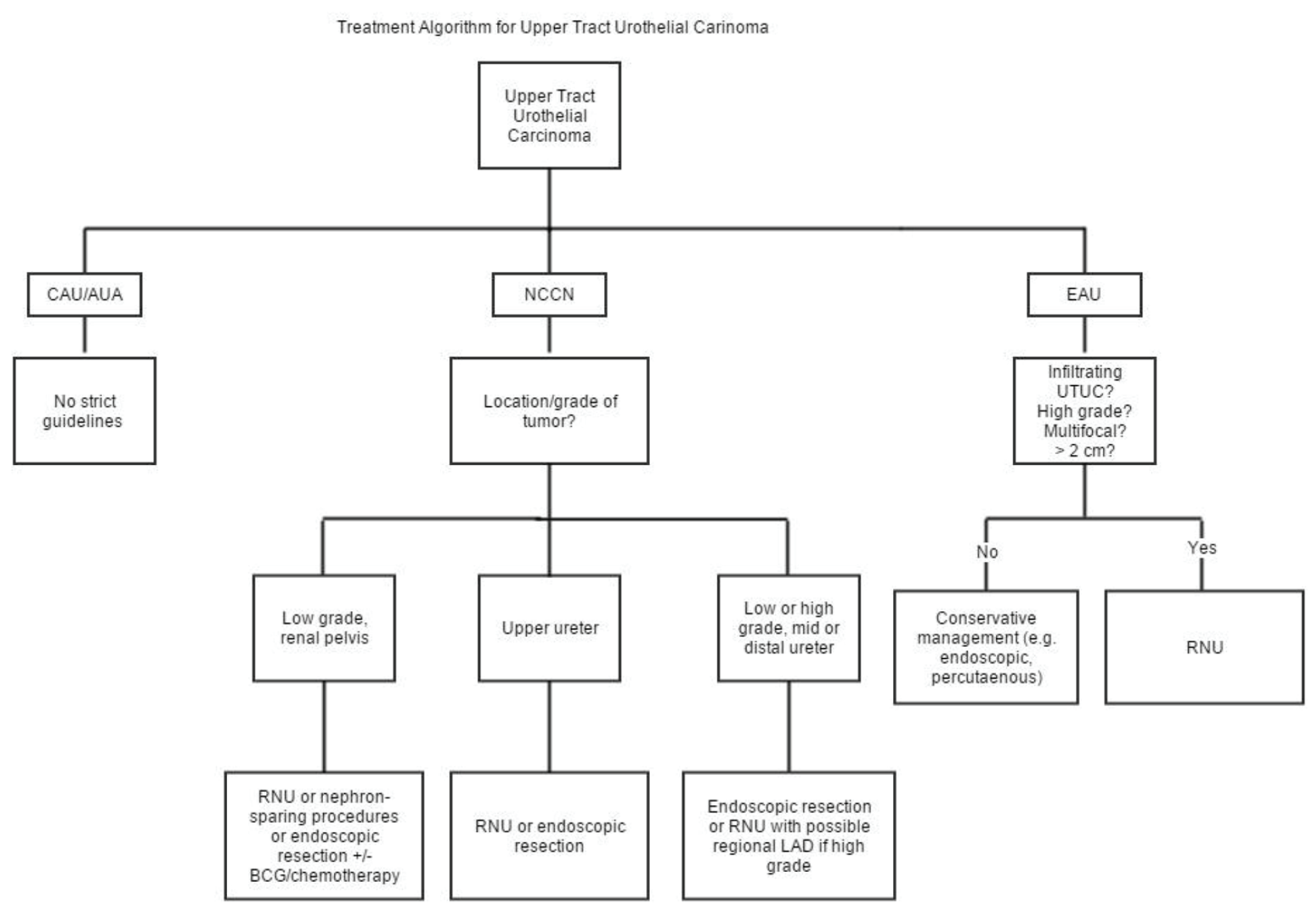

Figure 2. Treatment algorithm for UTUC per available guidelines.

\section{$[48,49]$.}

Lee et al address the impact of adjuvant chemotherapy in patients with UTUC and LVI following RNU. In this retrospective study of 344 patients, LVI was found to be an independent prognostic factor for cancer-specific survival and overall survival. Patients with T3-4 disease and LVI who were treated with adjuvant chemotherapy $(n=64)$ experienced improved cancer-specific survival (HR: $0.39, \mathrm{P}=0.028$ ) and overall survival (HR: $0.41, \mathrm{P}=0.031$ ). However, this survival benefit did not extend to patients with T1-T2 disease and LVI (HR: 1.89, $\mathrm{P}=0.604$ for cancer-specific survival and overall survival). A retrospective study by Lucca et al demonstrated similar results, with only patients harboring pT3/T4 N+ disease benefiting from adjuvant chemotherapy [50]. Another retrospective study by Kim et al, taking place between 2000 and 2013, investigated the impact of adjuvant gemcitabine plus cisplatin therapy following RNU in the setting of locally advanced disease $(n=65)$. In this study, $8 / 36(22.2 \%)$ patients who had undergone adjuvant chemotherapy experienced distant metastasis, compared with $6 / 29(20.7 \%)$ of those patients who had not received adjuvant therapy, with no statistically significant difference between the two arms of the study [48]. Hellenthal et al found similar results in their retrospective analysis of an international database of 1,390 patients, including 542 with high risk disease (PT3N0, PT4N0 and/or lymph node positive). Of these high risk patients 121 received adjuvant chemotherapy, with statistically significant survival benefit compared to those patients not receiving chemotherapy [49]. These studies and lack of current guidelines addressing adjuvant chemotherapy for UTUC underscore the importance of future RCTs.

\section{Radiation}

The role of radiation in adjuvant management of UTUC is poorly defined, with few retrospective non-randomized trials addressing this topic. Jwa et al evaluated clinical outcomes in patients with stage III/IV UTUC, with 36 patients undergoing adjuvant RT and 91 patients treated with surgery alone. With a median follow-up of 38.3 months, respective 3-year locoregional recurrence-free survival rates were $89 \%$ vs. $61 \%$ in the RT vs. non-RT groups $(\mathrm{P}=0.01)$ [48]. Another study by Fan et al demonstrated improved overall survival for patients receiving adjuvant chemoradiation following RNU vs. chemo- 
therapy and salvage radiation therapy [51]. Maulard-Durdux et al reported their experience with adjuvant radiation therapy given to 26 patients following complete resection of upper tract tumors, with overall 5-year survival rates and 5-year recurrence-free survival rates of $49 \%$ and $30 \%$, respectively, conferring no additional survival benefit compared to standard surgical therapy [52]. Further smaller trial results are mixed, and future analyses with robust sample sizes and prospective randomization are needed before definitive conclusions regarding adjuvant radiotherapy for UTUC can be reached.

\section{Follow-up}

NCCN guidelines for follow-up are uniform across all stages, regardless of primary treatment and use of adjuvant therapy. For all tumors NCCN recommends cystoscopy every 3 months for 1 year, then at increasing intervals thereafter. Following conservative endoscopic management, imaging of the upper tract collecting system is recommended at $3-12$ month intervals. CT scan, MRI, and chest X-ray are optional modalities for follow-up [19].

EAU provides grade $\mathrm{C}$ recommendations for follow-up of UTUC based upon treatment modality and tumor characteristics. For tumors treated with RNU, follow-up is recommended for at least 5 years following surgery. For non-invasive tumors treated with RNU recommendations are for yearly CT imaging. Cystoscopy and urine cytology should be obtained at 3 months and then yearly thereafter. Invasive tumors are followed more closely following RNU with cystoscopy/urinary cytology at 3 months and then yearly, as well as CTU every 6 months over 2 years and then yearly. Following conservative management of UTUC the EAU recommends follow-up for at least 5 years. Urine cytology and CTU is to be obtained at 3 and 6 months followed by yearly exams. Cystoscopy, ureteroscopy, and cytology in situ are recommended at 3 and 6 months, then every 6 months over 2 years, and then yearly thereafter.

The AUA currently does not provide specific guidelines for following these patients other than surveillance protocol used in patients with lower tract UC.

\section{Conclusions and Future Outlook}

Over the past decade, there has been a progressive move toward nephron preservation in treatment of renal cell carcinoma (RCC), initially provoked by data identifying a substantial increase in post radical nephrectomy chronic kidney disease (CKD), cardiovascular events, and overall mortality [53]. Broader application of this philosophy as it applies to treatment of UTUC has been a recent topic of debate in the urology community. Since urothelial carcinoma is known to be chemo-sensitive, nephron preservation is of interest with the potential need for platinum-based agents that are known to be nephrotoxic. However, we should be mindful that endoscopic resection of UTUC carries a much higher burden of local recurrence (20-85\%) [54] than does partial nephrectomy for RCC. Patients with UTUC require years of complicated and costly surveillance after local treatment and often require multiple serial endoscopic resections. With these caveats firmly in mind, studies by Simhan et al and Jeldres et al $[43,44]$ both support appreciation that nephron sparing approaches to lowgrade low-stage UTUC do not worsen cancer-specific mortality. Although these findings are encouraging, patients should be carefully selected for nephron salvage keeping with sound clinical judgment and adherence to published guidelines.

Guidelines on diagnosis and management of UTUC (Fig. 2) are lacking in current literature due to the rarity of the disease and lack of long-term data assessing its management. The EAU remains the primary provider of guidelines to date, but these guidelines are mainly based on small patient cohorts and retrospective studies with poor long-term follow-up data. Although the EAU advocates for more conservative surgical management, especially in cases of easily accessible, small low-grade tumors, there is a lack of strong (grade A) evidence necessary to clearly define a cohort of patients who would best be served with these treatment modalities. The state of current literature necessitates additional studies, preferably prospective in nature, with larger cohorts, and better long-term data. Questions still exist as to what size tumors can be successfully treated with less invasive measures, what clear role adjuvant therapy can play, how to best follow these patients, and when to proceed with radical treatment.

The accuracy of determining UTUC tumor stage and aggressiveness via noninvasive or minimally invasive tools remains controversial and necessitates further investigation. There exists a significant risk of under staging and undertreatment in conservatively managed upper urinary tract tumors [14]. In addition to the need for improved diagnostic modalities, further investigation into treatment options must be performed. LNU has yielded comparable results to open surgery with regard to cancer outcomes and also offers advantages in terms of morbidity and earlier convalescence [23]. However, since LNU may be selectively performed in low-risk patients (less tumor extent), it cannot be said with certainty that open nephroureterectomy and LNU have the same oncologic efficacy in high-risk patients. Long-term oncologic data in patients treated with LNU are required prior to acceptance of the procedure as standard of care for patients with high-grade and muscle-invasive UTUC. Further strategies and surveillance methods must be developed and validated to improve the outcomes in high-risk patients [15].

With the introduction of robotic technology, a trial assessing robot-assisted LNU vs. conventional LNU would be an additional point of future study with long-term follow-up data. Considering the challenges associated with performing an RCT in surgical practice it has been suggested that progressive surgical research will have to be reliant upon high-quality non-randomized trials [9].

Ultimately, when caring for the patient with UTUC, physicians must take into account the specific clinical characteristics of each individual patient with regard to renal function, the presence of co-morbidities, tumor location, grade, stage, and molecular marker status when determining the optimal treatment regimen. Strong consideration should be given to conservative management in the appropriately selected patients who agree to strict follow-up. 


\section{Funding}

None.

\section{Abbreviations}

AUA: American Urological Association; BCG: Bacillus Calmette-Guerin; CAU: Canadian Urological Association; CIS: carcinoma in situ; CTU: computed tomography urography; EAU: European Association of Urology; FISH: fluorescence in situ hybridization; LNU: laparoscopic nephroureterectomy; MRU: magnetic resonance urography; NCCN: National Comprehensive Cancer Network; PUNLMP: papillary urothelial neoplasia of low malignant potential; RCC: renal cell carcinoma; RNU: radical nephroureterectomy; TCC: transitional cell carcinoma; UTUC: upper tract urothelial carcinoma; WHO: World Health Organization

\section{References}

1. Munoz JJ, Ellison LM. Upper tract urothelial neoplasms: incidence and survival during the last 2 decades. J Urol. 2000;164(5):1523-1525.

2. Ploeg M, Aben KK, Kiemeney LA. The present and future burden of urinary bladder cancer in the world. World J Urol. 2009;27(3):289-293.

3. Garcia-Gonzalez R, Cuesta-Roca C, Garcia-Navas R, Gonzalez-Peramato P. [Upper urinary tract tumors pathology (ureter, pelvis and renal calyces)]. Arch Esp Urol. 2004;57(3):241-250.

4. Ristau BT, Tomaszewski JJ, Ost MC. Upper tract urothelial carcinoma: current treatment and outcomes. Urology. 2012;79(4):749-756.

5. Arancibia MF, Bolenz C, Michel MS, Keeley FX, Jr., Alken P. The modern management of upper tract urothelial cancer: surgical treatment. BJU Int. 2007;99(5):978981.

6. Cosentino M, Palou J, Gaya JM, Breda A, RodriguezFaba O, Villavicencio-Mavrich H. Upper urinary tract urothelial cell carcinoma: location as a predictive factor for concomitant bladder carcinoma. World J Urol. 2013;31(1):141-145.

7. Novara G, De Marco V, Dalpiaz O, Galfano A, Bouygues V, Gardiman M, Martignoni G, et al. Independent predictors of contralateral metachronous upper urinary tract transitional cell carcinoma after nephroureterectomy: multi-institutional dataset from three European centers. Int J Urol. 2009;16(2):187-191.

8. Audenet F, Yates DR, Cussenot O, Roupret M. The role of chemotherapy in the treatment of urothelial cell carcinoma of the upper urinary tract (UUT-UCC). Urol Oncol. 2013;31(4):407-413.

9. Roupret M, Babjuk M, Comperat E, Zigeuner R, Sylvester R, Burger M, Cowan N, et al. European guidelines on upper tract urothelial carcinomas: 2013 update. Eur Urol. 2013;63(6):1059-1071.
10. Rink M, Robinson BD, Green DA, Cha EK, Hansen J, Comploj E, Margulis V, et al. Impact of histological variants on clinical outcomes of patients with upper urinary tract urothelial carcinoma. J Urol. 2012;188(2):398-404.

11. Keeley FX, Kulp DA, Bibbo M, McCue PA, Bagley DH. Diagnostic accuracy of ureteroscopic biopsy in upper tract transitional cell carcinoma. J Urol. 1997;157(1):3337.

12. Iwaszko MR, Krambeck AE. Conservative management of upper tract transitional cell carcinoma. Indian J Urol. 2008;24(2):159-163.

13. Matin SF, Kamat AM, Grossman HB. High-frequency endoluminal ultrasonography as an aid to the staging of upper tract urothelial carcinoma: imaging findings and pathologic correlation. J Ultrasound Med. 2010;29(9):1277-1284.

14. Gadzinski AJ, Roberts WW, Faerber GJ, Wolf JS, Jr. Long-term outcomes of nephroureterectomy versus endoscopic management for upper tract urothelial carcinoma. J Urol. 2010;183(6):2148-2153.

15. Bird VG, Kanagarajah P. Surgical management of upper tract urothelial carcinoma. Indian J Urol. 2011;27(1):2-9.

16. Dasanu CA, Ong-Bacay A, Codreanu I. Newer developments in the therapeutics of the transitional cell carcinoma of renal pelvis. J Oncol Pharm Pract. 2012;18(1):97103.

17. Wein AJ, Kavoussi LR, Campbell MF. Campbell-Walsh urology / editor-in-chief, Alan J. Wein ; [editors, Louis R. Kavoussi ... et al.]. 10th ed. Philadelphia, PA: Elsevier Saunders; 2012.

18. Davis R, Jones JS, Barocas DA, Castle EP, Lang EK, Leveillee RJ, Messing EM, et al. Diagnosis, evaluation and follow-up of asymptomatic microhematuria (AMH) in adults: AUA guideline. J Urol. 2012;188(6 Suppl):2473-2481.

19. Simone G, Papalia R, Guaglianone S, Ferriero M, Leonardo C, Forastiere E, Gallucci M. Laparoscopic versus open nephroureterectomy: perioperative and oncologic outcomes from a randomised prospective study. Eur Urol. 2009;56(3):520-526.

20. Chen AA, Grasso M. Is there a role for FISH in the management and surveillance of patients with upper tract transitional-cell carcinoma? J Endourol. 2008;22(6):13711374.

21. Takahashi N, Glockner JF, Hartman RP, King BF, Leibovich BC, Stanley DW, Fitz-Gibbon PD, et al. Gadolinium enhanced magnetic resonance urography for upper urinary tract malignancy. J Urol. 2010;183(4):1330-1365.

22. Liang YY, Dai YP, Huang ZY, Zheng KL, Mei H. [Clinical analysis of 123 cases of transitional cell carcinoma (TCC) of upper urinary tract]. Ai Zheng. 2005;24(1):9194.

23. Park BH, Jeon SS. Endoscopic management of upper urinary tract urothelial carcinoma. Korean J Urol. 2013;54(7):426-432.

24. Grasso M. Ureteroscopic management of upper urinary tract urothelial malignancies. Rev Urol. 2000;2(2):116121.

25. Srirangam SJ, van Cleynenbreugel B, van Poppel H. 
Laparoscopic nephroureterectomy: the distal ureteral dilemma. Adv Urol. 2009:316807.

26. Lughezzani G, Sun M, Perrotte P, Shariat SF, Jeldres C, Budaus L, Alasker A, et al. Should bladder cuff excision remain the standard of care at nephroureterectomy in patients with urothelial carcinoma of the renal pelvis? A population-based study. Eur Urol. 2010;57(6):956-962.

27. Phe V, Cussenot O, Bitker MO, Roupret M. Does the surgical technique for management of the distal ureter influence the outcome after nephroureterectomy? BJU Int. 2011;108(1):130-138.

28. Zigeuner R, Pummer K. Urothelial carcinoma of the upper urinary tract: surgical approach and prognostic factors. Eur Urol. 2008;53(4):720-731.

29. Ubrig B, Boenig M, Waldner M, Roth S. Transurethral approach to the distal ureter in nephroureterectomy: transurethral extraction vs. "pluck" technique with long-term follow-up. Eur Urol. 2004;46(6):741-747.

30. Palou J, Caparros J, Orsola A, Xavier B, Vicente J. Transurethral resection of the intramural ureter as the first step of nephroureterectomy. J Urol. 1995;154(1):43-44.

31. Shariat SF, Godoy G, Lotan Y, Droller M, Karakiewicz PI, Raman JD, Isbarn H, et al. Advanced patient age is associated with inferior cancer-specific survival after radical nephroureterectomy. BJU Int. 2010;105(12):16721677.

32. Chromecki TF, Ehdaie B, Novara G, Pummer K, Zigeuner R, Seitz C, Pycha A, et al. Chronological age is not an independent predictor of clinical outcomes after radical nephroureterectomy. World J Urol. 2011;29(4):473-480.

33. Matsumoto K, Novara G, Gupta A, Margulis V, Walton TJ, Roscigno M, Ng C, et al. Racial differences in the outcome of patients with urothelial carcinoma of the upper urinary tract: an international study. BJU Int. 2011;108(8 Pt 2):E304-309.

34. Isbarn H, Jeldres C, Shariat SF, Liberman D, Sun M, Lughezzani G, Widmer $\mathrm{H}$, et al. Location of the primary tumor is not an independent predictor of cancer specific mortality in patients with upper urinary tract urothelial carcinoma. J Urol. 2009;182(5):2177-2181.

35. Miyake H, Hara I, Gohji K, Arakawa S, Kamidono S. The significance of lymphadenectomy in transitional cell carcinoma of the upper urinary tract. Br J Urol. 1998;82(4):494-498.

36. Komatsu H, Tanabe N, Kubodera S, Maezawa H, Ueno A. The role of lymphadenectomy in the treatment of transitional cell carcinoma of the upper urinary tract. J Urol. 1997;157(5):1622-1624.

37. Mills IW, Laniado ME, Patel A. The role of endoscopy in the management of patients with upper urinary tract transitional cell carcinoma. BJU Int. 2001;87(2):150-162.

38. Elliott DS, Segura JW, Lightner D, Patterson DE, Blute ML. Is nephroureterectomy necessary in all cases of upper tract transitional cell carcinoma? Long-term results of conservative endourologic management of upper tract transitional cell carcinoma in individuals with a normal contralateral kidney. Urology. 2001;58(2):174-178.

39. Wolf JS, Jr. Are we underutilizing minimally invasive approaches for upper tract urothelial carcinoma? Urol On- col. 2009;27(1):75-80.

40. Adamis S, Varkarakis J. Minimally invasive approach in the management of upper- urinary-tract tumours. Scand J Urol Nephrol. 2011;45(6):381-387.

41. Roupret M, Hupertan V, Traxer O, Loison G, ChartierKastler E, Conort P, Bitker MO, et al. Comparison of open nephroureterectomy and ureteroscopic and percutaneous management of upper urinary tract transitional cell carcinoma. Urology. 2006;67(6):1181-1187.

42. Cutress ML, Stewart GD, Zakikhani P, Phipps S, Thomas BG, Tolley DA. Ureteroscopic and percutaneous management of upper tract urothelial carcinoma (UTUC): systematic review. BJU Int. 2012;110(5):614-628.

43. Simhan J, Smaldone MC, Egleston BL, Canter D, Sterious SN, Corcoran AT, Ginzburg S, et al. Nephron-sparing management vs radical nephroureterectomy for low- or moderate-grade, low-stage upper tract urothelial carcinoma. BJU Int. 2014;114(2):216-220.

44. Jeldres C, Lughezzani G, Sun M, Isbarn H, Shariat SF, Budaus L, Lattouf JB, et al. Segmental ureterectomy can safely be performed in patients with transitional cell carcinoma of the ureter. J Urol. 2010;183(4):1324-1329.

45. Wang AJ, Goldsmith ZG, Neisius A, Astroza GM, Oredein-McCoy O, Iqbal MW, Simmons WN, et al. Increasing dwell time of mitomycin $\mathrm{C}$ in the upper tract with a reverse thermosensitive polymer. J Endourol. 2013;27(3):288-293.

46. Audenet F, Traxer O, Bensalah K, Roupret M. Upper urinary tract instillations in the treatment of urothelial carcinomas: a review of technical constraints and outcomes. World J Urol. 2013;31(1):45-52.

47. Edge SB, Compton CC. The American Joint Committee on Cancer: the 7th edition of the AJCC cancer staging manual and the future of TNM. Ann Surg Oncol. 2010;17(6):1471-1474.

48. Kim TS, Oh JH, Rhew HY. The efficacy of adjuvant chemotherapy for locally advanced upper tract urothelial cell carcinoma. J Cancer. 2013;4(8):686-690.

49. Hellenthal NJ, Shariat SF, Margulis V, Karakiewicz PI, Roscigno M, Bolenz C, Remzi M, et al. Adjuvant chemotherapy for high risk upper tract urothelial carcinoma: results from the Upper Tract Urothelial Carcinoma Collaboration. J Urol. 2009;182(3):900-906.

50. Lucca I, Kassouf W, Kapoor A, Fairey A, Rendon RA, Izawa JI, Black PC, et al. The role of adjuvant chemotherapy for lymph node-positive upper tract urothelial carcinoma following radical nephroureterectomy: a retrospective study. BJU Int. 2014.

51. Fan KH, Chen YC, Leung WM, Chuang CK, Pang ST, Hong JH. Adjuvant and salvage radiotherapy for urothelial cell carcinoma of the upper urinary tract: experience in a single institution. Chang Gung Med J. 2012;35(3):247254.

52. Chen B, Zeng ZC, Wang GM, Zhang L, Lin ZM, Sun LA, Zhu TY, et al. Radiotherapy may improve overall survival of patients with T3/T4 transitional cell carcinoma of the renal pelvis or ureter and delay bladder tumour relapse. BMC Cancer. 2011;11:297.

53. Go AS, Chertow GM, Fan D, McCulloch CE, Hsu CY. 
Chronic kidney disease and the risks of death, cardiovascular events, and hospitalization. N Engl J Med. 2004;351(13):1296-1305.
54. Bagley DH, Grasso M, 3rd. Ureteroscopic laser treatment of upper urinary tract neoplasms. World J Urol. 2010;28(2):143-149. 